\title{
L'ESTUATRE DE LA RANCE
}

\section{SEDMIENTOLOGIE ET AMENAGEMENT}

\author{
C. BONNOT-COURTOIS. URA 904 CNRS. Laboratoire de Géomorphologie.
}

\section{CARTE SEDIMENTOLOGIQUE DU BASSIN MARITIME}

La carte sédimentologique de l'estuaire de la Rance a été établie à partir de l'analyse granulométrique d'environ 150 prélèvements de sédiments superficiels, effectués en 1994 dansd l'ensemble du bassin maritime et permet de mettre en évidence les gradients granulométriques longitudinaux et transversaux. A l'embouchure de l'estuaire proche de l'usine, le chenal est tapissé de cailloutis et de sables grossiers, mais le fond des anses est caractérisé par une sédimentation fine de sables vaseux. Au fur et à mesure que l'on va vers l'amont de la retenue, les sédiments deviennent de plus en plus fins, les sables grossiers et moyens faisant place aux sables fins puis à des silts et de la vase pratiquement pure à partir de l'étroiture de Port Saint-Jean/Port Saint-Hubert. La répartition des sédiments superficiels suit la distribution des courants de marée qui sont atténués depuis la mise en service de l'usine mais qui sont largement guidés par la géomorphologie de la ria. Les parties resserrées où les courants conservent une certaine vitesse comprennent des sables moyens et grossiers, tandis que les secteurs évasés des anses ou la partie plus amont du bassin maritime sont des zones d'annulation des courants où se déposent des vases de plus en plus fines. L'évolution granulométrique avec tendance à l'affinement est mise en évidence d'aval en amont de l'estuaire et également depuis le chenal central jusque dans les parties les plus internes et les plus hautes des anses qui sont colonisées par la végétation halophile.

L'exploitation de l'usine a entrainé des modifications du régime hydrologique de l'estuaire, essentiellement par prolongation des étales qui peuvent durer deux heures à l'intérieur du bassin au lieu d'une dizaine de minutes côté mer, et par réduction du marnage qui n'est plus que d'environ $9 \mathrm{~m}$ dans le bassin de retenue. Ces modifications hydrologiques ont des conséquences sur l'équilibre sédimentologique de l'estuaire. La prolongation des étales dans le bassin par rapport à la mer ouverte laisse aux particules en suspension le temps d'atteindre les fonds et d'y sédimenter. La réduction du marnage et en particulier le fait que le niveau du bassin ne descende que très exceptionnellement en-dessous de $4 \mathrm{~m}$ entraine une diminution des courants de jusant qui sont moins efficaces pour éroder les fonds et évacuer vers l'extérieur les matières en suspension. L'annulation du courant pendant les étales prolongées et l'atténuation du jusant favorisent théoriquement la sédimentation, particulièrement dans les zones abritées des anses et sur les berges de 1'estuaire ainsi que dans les secteurs calmes à l'amont du bassin maritime. La comparaison de photographies aériennes réalisées sur l'ensemble du bassin en 1953 et en 1985 souligne les changements intervenus après 20 ans de fonctionnement sous un régime de marée modifié. La surface des zones exondables a été notablement réduite et certains secteurs des berges ont été envasés. 


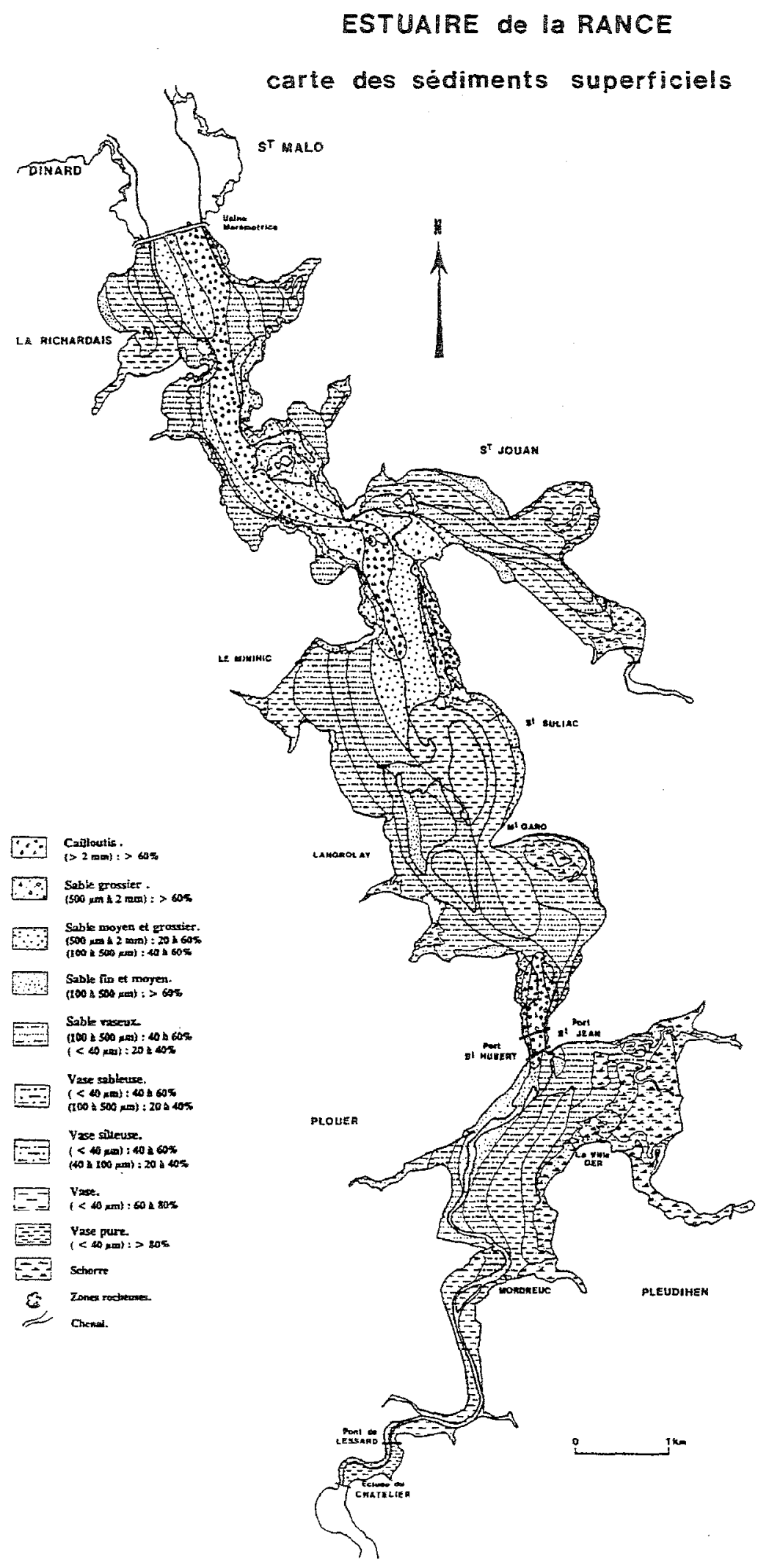




\section{DNVASEMENT DANS LA PARTIE AMONT DU BASSIN MARITME}

Depuis la mise en service de l'usine en 1967, les dépôts de vase à l'intérieur du bassin de retenue n'ont pas posé de problème majeur pendant près de 25 ans, la bathymétrie. ne subissant que des modifications mineures liées essentiellement aux déplacements des chenaux. Cependant, entre 1989 et 1991, la partie amont du bassin maritime, située à proximité de l'écluse du Châtelier a connu une augmentation de la sédimentation à tel point que des dragages ont dû être réalisés afin de maintenir des profondeurs du chenal suffisantes pour la navigation. Cette augmentation de la sédimentation est liée à un déficit pluviométrique important pendant trois années consécutives qui a provoqué une réduction importante des débits de la Rance. La forte diminution des apports fluviatiles au niveau de l'écluse du Châtelier a largement favorisé la remontée du bouchon vaseux et donc de la zone préférentielle de sédimentation à l'amont du bassin maritime, aboutissant au dépôt de vases très fines et peu consolidées dans le chenal. L'année 1992 a été marquée par une amélioration de la pluviométrie qui a dépassé la moyenne annuelle dans l'ensemble du bassin versant de la Rance, ce qui a permis de rétablir des débits fluviatiles suffisants pour repousser la zone de très forte turbidité vers l'aval de l'estuaire ne rendant plus nécessaires les dragages d'entretien du chenal.

Des expériences de suivi des rejets de dragages menées en collaboration avec EDF, Usine marémotrice de la Rance, ont été menées dans toute la partie amont du bassin maritime comprise entre Port Saint-Jean et l'écluse du Châtelier pendant différents cycles de marée. La dynamique des matières en suspension ainsi que les variations de salinité ont lieu essentiellement dans la partie amont la plus étroite de l'estuaire entre l'écluse du Châtelier et Mordreuc. L'étroitesse du chenal de navigation interdit le passage d'engins de dragage trop importants ; leur puissance est de ce fait limitée et la localisation du point de rejet reste très proche $(800$ à $1000 \mathrm{~m})$ de l'écluse du Chatelier. Même si le rejet n'a lieu que pendant la vidange du bassin, l'extension du panache turbide vers l'aval ne dépasse pas la zone amont de Mordreuc en raison de la faible vitesse des courants de jusant, et la remontée du flot remporte vers l'amont la majeure partie des matières en suspensions. D'autre part, des expérimentations de chasse hydraulique ont montré que la remise en suspension des vases et la dispersion du panache turbide étaient plus efficaces qu'au cours d'un dragage n'utilisant que le courant de jusant. Les conditions optimales de mise en oeuvre des chasses hydrauliques doivent permettre une bonne remise en suspension des sédiments ainsi qu'un transport des matières en suspension suffisamment loin vers l'aval en un temps assez court ayant que le flot suivant ne retransporte les suspensions vers l'amont. En cas de sécheresse, une meilleure gestion du plan d'eau à l'amont de l'écluse devrait permettre de reconstituer le potentiel de chasse hydraulique de la Rance fluviale et ainsi de rétablir des crues artificielles du même ordre de grandeur que les crues naturelles de la Rance pour évacuer les matières en suspension vers l'aval de l'estuaire. 

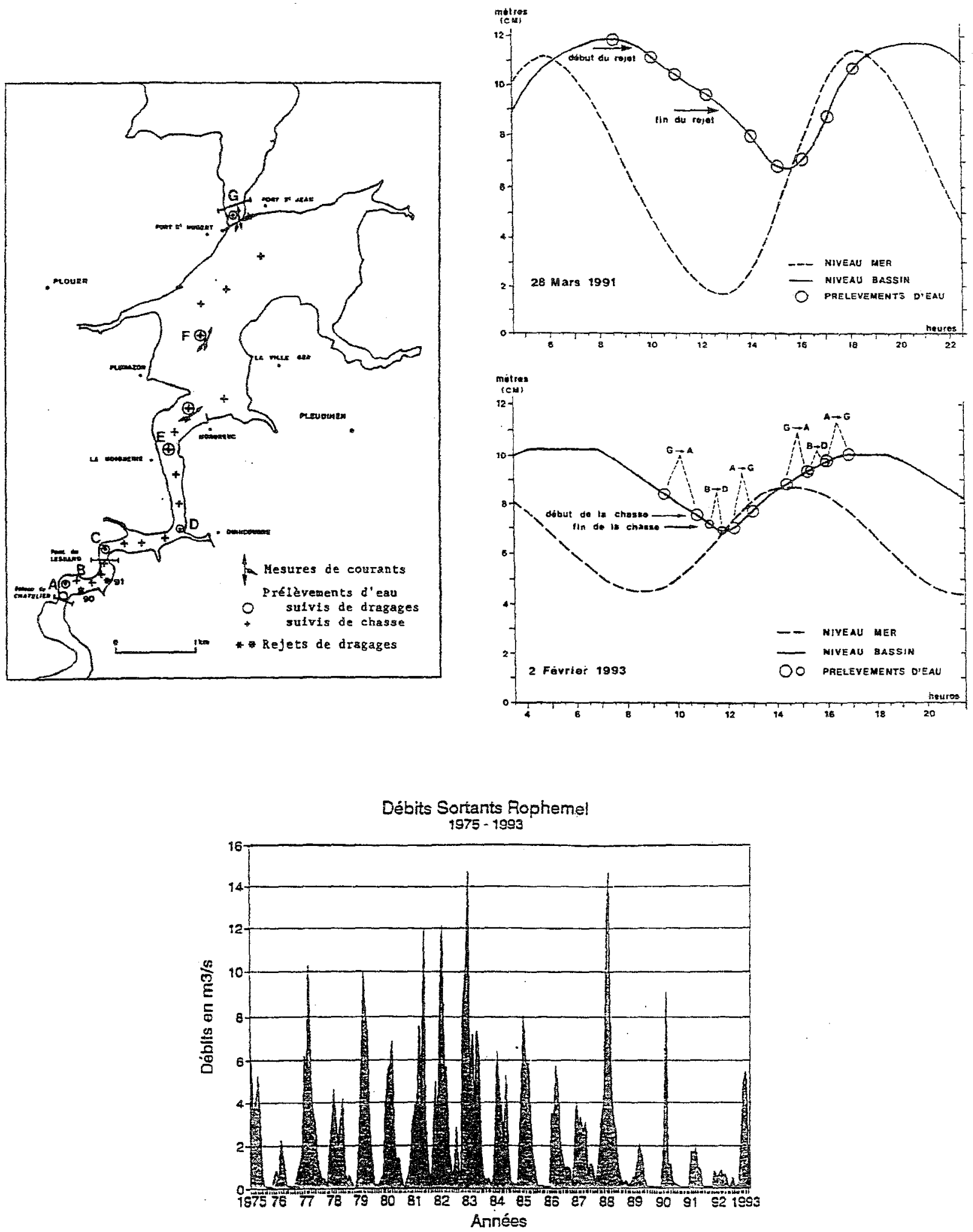

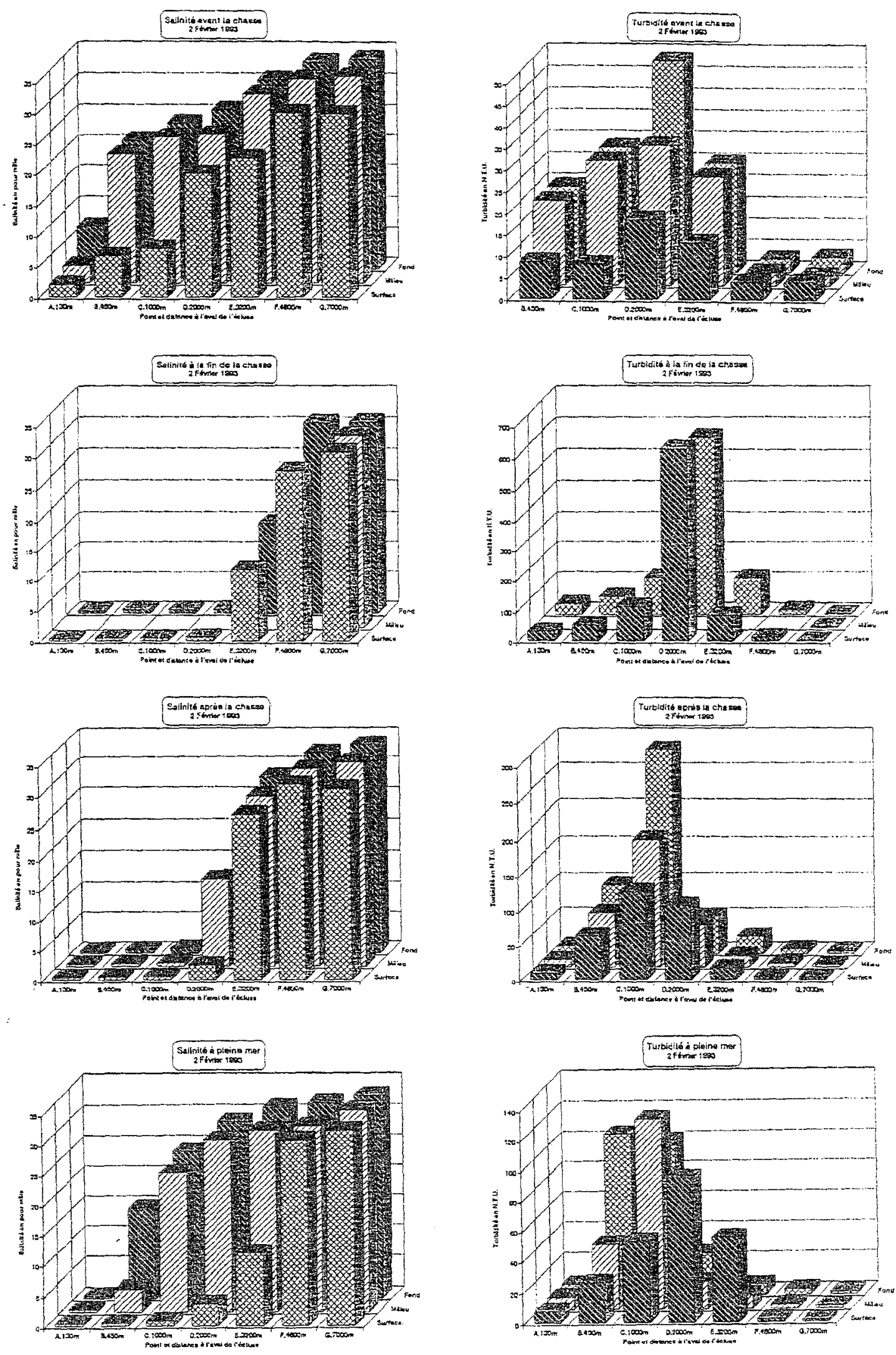

Répartition de 1 a salinité

Répattition de la turbidité

au couts de la chasse hyoraulique expérimentale 